Sociologie et sociétés

\title{
Travail en miettes, activité, activation
}

À propos de quelques déplacements paradigmatiques de la sociologie du travail depuis les années 1960

Piecemeal work, activity, activation About a few paradigmatic shifts in sociology since the 1960s

\section{Alexander Neumann}

Volume 48, numéro 1, printemps 2016

Le travail au prisme de l'activité

Work through the lens of activity

URI : https://id.erudit.org/iderudit/1036883ar

DOI : https://doi.org/10.7202/1036883ar

Aller au sommaire du numéro

\section{Éditeur(s)}

Les Presses de l’Université de Montréal

\section{ISSN}

0038-030X (imprimé)

1492-1375 (numérique)

\section{Découvrir la revue}

Citer cet article

Neumann, A. (2016). Travail en miettes, activité, activation : à propos de quelques déplacements paradigmatiques de la sociologie du travail depuis les années 1960. Sociologie et sociétés, 48(1), 59-79.

https://doi.org/10.7202/1036883ar

\section{Résumé de l'article}

La discussion qui se déploie actuellement autour du concept d'activité nous invite à interroger le développement théorique de la sociologie du travail, depuis les travaux fondateurs des années 1950 et 60, à travers plusieurs tournants paradigmatiques successifs, jusqu'aux enjeux présents. La lecture théorique que nous poursuivons est donc celle d'un détachement progressif du travail pratiqué, qui aboutit à une focalisation sur l'organisation, du moins dans les courants les plus visibles de l'espace académique et public. Dans ce cadre, nous comprenons la notion d'activité à la fois comme un effort de recentrer le regard sociologique sur le travail en actes qui est exercé et vécu par les salariés, et comme une conception alternative à la vision d'un travail sans qualité (sous sa forme prétendument interchangeable, cognitive ou numérique). 


\title{
Travail en miettes, activité, activation
}

\author{
À propos de quelques déplacements paradigmatiques \\ de la sociologie du travail depuis les années 1960
}

\section{ALEXANDER NEUMAN N}

Centre d'études sur les médias, les techniques et

I'internationalisation (CEMTI)

UFR Culture et communication

Université Vincennes

Courriel: aneumanno2@univ-paris8.fr

\section{INTRODUCTION}

T A Discussion Qui SE DÉPloie AUTOUR DU CONCEPT D’ACTIVITÉ nous invite à ـinterroger le développement théorique de la sociologie du travail, depuis les travaux fondateurs des années 1950 et 1960, à travers plusieurs tournants paradigmatiques successifs qui déplacent les leitmotivs sociologiques, jusqu'aux enjeux présents.

À la thèse durkheimienne du travail comme donnée positive qui devait engendrer une solidarité morale à travers la division sociale du travail, Friedmann opposa son constat d'un travail en miettes, au vu d'un processus de rationalisation historique (Friedmann, 1964). Par la suite, Horst Kern et Michael Schumann ont repris cette question en titre d'un livre: La fin de la division sociale du travail? (Kern et Schumann, 1984). Alain Touraine, issu de la tradition friedmannienne, a proposé une analyse de la conscience ouvrière qui s'éloigne peu à peu du rapport au poste de travail pour considérer des échelles d'organisation plus vastes, y compris politicio-administratives. Il a fini par délaisser la notion de travail au cours des années 1980 et 1990, pour la subsumer à sa sociologie de l'action. Dans le même temps, Ulrich Beck a inscrit la problématique du travail dans le paradigme du risque. Pareille approche a pu favoriser la conception quantitative et d'un travail sans qualités (Sennet, 2000), abordé sous 
l'angle de La gestion des risques (Castel, 2011). La querelle publique au sujet de la fin du travail a illustré le versant philosophique de cette problématique vers la fin des années 1990. Le dernier stade de cette évolution sociologique est la proéminence du principe d'activation des demandeurs d'emploi qui s'étend sur l'intégralité du marché de l'emploi à travers le principe de flexicurité et sa vision macroanalytique.

La lecture théorique que nous poursuivons est donc celle d'un détachement progressif du travail pratiqué, qui aboutit à une focalisation sur l'organisation, du moins dans les courants les plus visibles de l'espace didactique et public. Dans ce cadre, nous comprenons la notion d'activité à la fois comme un effort de recentrer le regard sociologique sur le travail en actes qui est exercé et vécu par les salariés, et comme une conception alternative à la vision d'un travail sans qualités. Enfin, nous inscrivons la renaissance récente d'une discussion sociologique autour du travail et de l'activité, sur le plan conceptuel, dans le mouvement du retour d'une conception critique.

\section{TRAVAIL EN MIETTES, AUTOMATISME SOCIAL, CONSCIENCE OUVRIÈRE}

Au terme du cycle fondateur de la sociologie du travail, marqué par un nouveau mouvement de rationalisation historique depuis l'après-guerre, Georges Friedmann conceptualise les limites du management taylorien et de sa décomposition du processus de travail en tâches. Il constate alors «la mécanisation de la rationalisation» et des tâches semi-qualifiées qui l'appuient dans tous les secteurs (l'industrie, le commerce, les bureaux et l'agriculture). La spécialisation des postes engage un éclatement des tâches et des modalités d'apprentissage dégradées, appauvries. Ceci se fonde sur des observations empiriques partielles, dans des conditions d'accès difficiles, comme cela est toujours le cas dans les entreprises. Le contraste saisissant entre les expériences que recueille Friedmann et les principales thèses de Durkheim pousse le sociologue du travail à une clarification théorique dont le caractère tranché ne doit pas être sousestimé: «Si Durkheim avait vécu, il aurait été obligé, afin de maintenir dans sa pureté la thèse de la solidarité organique, de considérer comme «anormales» la plupart des formes que le travail a prises dans nos sociétés» (Friedmann, 1964: 144). Les enquêtes qu'il vient de réaliser montrent que l'interdépendance mécanique ne suscite pas la solidarité morale, mais plutôt une variété de situations que seul le syndicalisme ouvrier semble en mesure d'unifier de manière constructive, et se manifeste comme le véritable lieu de la solidarité. Il conclut: «Emile Durkheim a situé haut, trop haut sans doute, et loin des faits la plupart des exemples à l'aide desquels il a pensé son système» (Friedmann, 1964: 146). Nous notons au passage que le thème de la rationalisation est au cœur des écrits de Max Weber rassemblés dans Économie et société, dont la première publication française n'est cependant parue qu'au début des années 1970 (Weber, 1971).

La remise en question conceptuelle des thèmes durkheimiens s'inscrit dans le cadre plus large du Traité de sociologie du travail (Friedmann et Naville, 1961). Pendant que Friedmann souligne la spécialisation des tâches, qui aboutit à des emplois peu ou semi-qualifiés dans tous les domaines, y compris les supermarchés et les bureaux, 
Naville (1957b) pointe la perspective plus vaste d'un automatisme social où toutes les activités seraient automatisées, phénomène qu'il observe directement lors de la robotisation de l'industrie, qui est aujourd'hui illustré à travers l'informatisation.

Sur la base d'une observation de mille entreprises françaises, Naville montre que la rationalisation progressive et étendue qui touche les grands secteurs industriels aboutit à une situation, où le rapport individuel au travail se trouve complètement déconnecté des schèmes globaux de l'organisation du travail. Le temps de production peut atteindre 24 heures sur 24 et se trouve dissocié du temps travaillé, réparti sur plusieurs équipes successives (Naville, 1957b). Le sociologue retrace ensuite l'effet de propagation de ce principe au-delà de l'industrie, dans le commerce et les bureaux (Naville, 1964). Le temps des activités de service tend aujourd'hui à suivre ce mouvement, avec des hotlines accessibles en permanence et des commerces dont l'amplitude d'ouverture approche les 24 heures sur 24 également, dimanche compris. La spécialisation des tâches se voit ainsi doublée par une dissociation temporelle qui fait que peu des salariés travaillent sur le même lieu en même temps. Il en résulte, sur le plan théorique, une distinction nette entre emploi, travail et activité (Rolle, 1997).

Sur le plan théorique, la conséquence de la dynamique de rationalisation est l'abolition du modèle durkheimien qui avait parié sur la solidarité organique devant résulter de la division sociale du travail, conséquence que tirent solidairement Friedmann et Naville. Il est vrai que Friedmann part du modèle de l'artisanat (par exemple du travail d'un peintre) pour juger l'appauvrissement des tâches, même lorsqu'il aborde la rationalisation taylorienne du travail ouvrier (Friedmann, 1964: 158; Bidet, 2011). Prenant appui sur Naville, Friedmann admet qu'une spécialisation qui est appuyée sur une grande compétence (celle du chirurgien par exemple) diffère fondamentalement de celle d'un ouvrier spécialisé, réduit à une tâche monotone aussitôt obtenu le brevet (Friedmann, 1964: 161). Alors que Friedmann cherche à valoriser le travail en actes, à travers la polyvalence ou la qualification, Naville développe une théorie critique plus générale de l'aliénation et du salariat à la suite de Marx, qui l'éloigne de l'analyse circonscrite de l'activité de travail.

Si Friedmann mobilise de nombreux éléments empiriques, centrés sur la spécialisation du travail des ouvriers et des employés, pour contredire les thèses durkheimiennes, sans pour autant présenter une théorie générale, Naville penche vers la formulation d'une théorie critique du salariat qui sollicite les situations de travail plutôt comme exemples ou illustrations (Neumann, 2010). Les 5 tomes du Nouveau Léviathan à travers lesquels Naville déploie sa conception théorique s'organisent autour du concept clé du «travail abstrait», emprunté à Marx mais dont l'usage est marginal dans le marxisme de l'époque. Le concept signale l'importance du caractère socialisé du travail, qui se réalise à travers le marché, et la subsomption de toutes les activités au principe du capital qui les intègre comme étant du capital variable, à commencer par le travail lui-même (Naville, 1957a).

Cette constellation sociologique décrit un écart entre la recherche empirique et l'effort de conceptualisation critique qui ne sera sans doute jamais comblé intégrale- 
ment, comme semble déjà l'indiquer le débat épistémologique des années 1960 (Frisby, 1974). Dans l'entre-deux de cette polarité, Alain Touraine a tenté de reconstruire une théorie de la conscience ouvrière, d'abord en partant des situations de production industrielle et des différents rapports au travail y correspondant, ensuite en élargissant le champ d'observation aux organisations et administrations à l'échelle de toute la société. Contrairement à Friedmann, Touraine n'entend pas se limiter au travail ouvrier concret, mais le place dans un «système social» du travail en s'intéressant au mode d'organisation de l'entreprise. Cela lui vaut une critique assez verte du «maître», qui lui reproche de s'être lancé dans des généralisations abstraites et hâtives, au lieu de progresser par déduction à partir des observations empiriques (Düll, 1975: 119).

Depuis ses travaux sur les usines Renault, Touraine avait discerné trois systèmes, connus sous le nom de phases $\mathrm{A}, \mathrm{B}$ et $\mathrm{C}$ (Touraine, 1955). L'auteur signale à plusieurs reprises que les trois systèmes ne constituent pas des étapes historiques ou chronologiques (Touraine, 1999: 200), mais qu'ils peuvent coexister dans le temps. L'auteur se défend de prôner un déterminisme technique, mais les formes de la conscience semblent bel et bien découler de la nature des entreprises dont les salariés dépendent. La «classe ouvrière» paraît donc se constituer à partir de la situation de travail. Dans un commentaire critique, Düll souligne «l'ouvriérisme sociologique» de l'équipe réunie par Georges Friedmann, à laquelle Touraine participe, et qui voit la classe ouvrière comme une donnée sociale qui peut être éprouvée sans détour, à travers les enquêtes de terrain (Düll, 1975: 110).

Cet arrière-fond théorique pèse encore dans la description que Touraine fait de la «conscience ouvrière», qui semble largement influencée par l'appareil de production et ses techniques. À nouveau, nous sommes placé devant des machines et un travail immédiat. L'auteur souligne que l'objet principal de son ouvrage est de montrer «les transformations de la conscience ouvrière liées à l'évolution technique» (Touraine, 1966: 28). Dans son ouvrage dédié à la «conscience ouvrière», Touraine affirme alors le principe suivant: "Il est nécessaire et possible de définir la conscience de classe au travail» (nous soulignons, Touraine, 1966: 113).

Selon les définitions tourainiennes, le travailleur entretient un rapport subjectif immédiat à la production, tandis que chez Marx, le salarié est d'abord défini comme étant la partie variable de la valorisation capitaliste, produisant des marchandises sans qualité, par un travail sans qualité (Marx, 2015). À partir du moment où l'interprétation « ouvriériste» met de côté les médiations sociales qui découlent du travail salarié, hormis le cadre organisationnel de l'entreprise, il est logique que Touraine remplace in fine la sociologie du travail par une sociologie de l'action, afin d'atteindre le niveau théorique de la société comme un «ensemble de formes sociales» (Touraine, 2000: 41). L'auteur a d'abord cherché à répondre au dilemme d'un travail en entreprise qui ne rencontre pas immédiatement la société dans son ensemble, en centrant son argumentation sur la notion de «rationalisation». Ce terme induit un renversement de perspective qui délaisse le travail ouvrier au profit de l'entreprise (en tant qu'organisation): "Plus la rationalisation se développe et s'élève vers les niveaux supérieurs de 
fonctionnement de l'entreprise, plus aussi l'ensemble des problèmes sociaux du travail apparaît comme la lutte pour le contrôle des instruments et des résultats du travail rationalisé» (Touraine, 1999: 195). Pareil déplacement de terrain permet à Touraine de lier, ensuite, l'organisation sociale à l'action politique: "Plus la production s'organise, plus le sujet historique devient collectif, plus le pouvoir se concentre et donc plus les conflits sociaux deviennent politiques» (Touraine, 1999: 212). Comme ce constat contredit in fine l'idée que la conscience ouvrière naîtrait directement de la situation de travail concrète, Touraine tente de combler le manque théorique qui en résulte, en considérant la conscience de classe sur le plan politique et culturel. Celle-ci ne peut se manifester qu'à travers un conflit qui se joue sur le plan global de la société, autour de la définition du bien commun, et en opposition aux intérêts privés (Touraine, 1999: 119). Nous constatons qu'il s'agit d'une définition très proche de ce qu'Arendt nomme l'espace public. Le concept tourainien de «conscience» hésite ainsi, entre le travail ouvrier, la conscience de classe et l'espace du politique, sans jamais retrouver un équilibre théorique. La notion de «conscience» se démultiplie pour laisser place à une polysémie: conscience impuissante, prolétarienne, ouvrière, de classe, etc., se succèdent et existent parfois simultanément dans les textes. Ces notions se concurrencent au sein d'un jeu de connotations dont on voit mal l'unité théorique. Par la suite, Touraine résout ce problème en abandonnant le concept de travail salarié à travers une «sociologie de la liberté et de l'action historique» (Touraine, 1999: 121) qui se substitue à la sociologie du travail. Il précise ainsi que le contexte historique au sein duquel il situait l'action collective et le mouvement social est devenu caduc, face à une société «postindustrielle» marquée par la communication et l'information qui aurait définitivement supplanté le travail salarié (Touraine, 1999: 14). Sa réflexion part des enjeux liés à «la transformation sociale» et suppose l'affranchissement des contraintes et représentations qui pèsent sur le travail salarié; en l'absence d'une transition théorique de l'un à l'autre, c'est-à-dire du travail à la politique, le travail salarié disparaît simplement du champ d'investigation, alors que la sociologie de l'action s'intéresse aux modes d'organisation et de communication qui ont été engendrés par le travail d'un point de vue historique, en tant qu'autoproduction de la société. Dans les années 2000, Touraine a présenté cette évolution théorique comme une succession historique de paradigmes, où l'on passe du paradigme «militaropolitique» du $19^{\mathrm{e}}$ siècle au paradigme industriel et économique du $20^{\mathrm{e}}$, pour finir sur un paradigme culturel correspondant au $21^{\mathrm{e}}$ siècle naissant, davantage centré sur l'œuvre que sur le travail (Touraine, 2005: 9). Dès lors, il est cohérent qu'il utilise surtout les notions de nation et d'opinion publique en lieu et place du langage du travail et de la conscience ouvrière.

L'évolution de Touraine décrit une tendance plus générale de la sociologie du travail vers le leitmotiv de l'organisation, et la transformation de la conception du travail en une théorie de l'action. Ce déplacement de terrain peut aussi être illustré par les travaux de Michel Crozier, dont le point de départ n'est pas éloigné de Touraine, car tous deux font partie des fondateurs de la revue Sociologie du travail en 1959. 
Crozier s'appuie ensuite sur des enquêtes réalisées dans des administrations publiques françaises pour développer une théorie de la bureaucratie qui fonde à son tour sa conception de l'action: "Une théorie de la bureaucratisation constitue donc forcément un cas particulier d'une théorie plus générale des organisations, qui devrait constituer ellemême, à son tour, l'élément essentiel d'une sociologie de l'action valable pour l'étude de la société globale» (nous soulignons, Crozier, 1963: 20). Tout comme la conceptualisation de Touraine s'éloigne de son objet empirique premier, la situation de travail en usine, le développement théorique de Crozier se sert du cas particulier d'administrations françaises pour aller vers une théorie globale de l'action. Pour ce faire, l'auteur se réfère aux travaux classiques de Max Weber, qui avait cependant mis en garde ses successeurs, en montrant qu'au fur et à mesure qu'une théorie devient globale et conceptuellement pure, elle s'éloigne de plus en plus de la réalité empiriquement observable (Weber, 1922). Dans ses écrits ultérieurs, Crozier ne part plus de la description minutieuse d'administrations ou d'un autre contexte de travail, mais directement du point de vue global de l'organisation, qui s'exprime dans le titre d'un livre publié dans l'après-68, La société bloquée (Crozier, 1970). La grève générale de 1968 n’y est pas abordée à partir de l'expérience du travail, mais à partir de l'organisation générale de la société française, jusqu'au refus par l'auteur du référendum de 1969 en faveur de la participation des salariés, proposé par le président de Gaulle. Sur un plan plus théorique, le modèle de l'organisation est assumé en tant que construction sociologique universelle, dont la transposition à l'ensemble des situations sociales se trouve argumentée dans L'acteur et le système (Crozier, Friedberg, 1977).

Rétrospectivement, le fait que cette évolution conceptuelle coïncide avec l'abandon ou la prédominance de positions critiques envers toutes les variations du marxisme sociologique, prégnantes chez Friedmann et Naville lors de la préparation du traité de sociologie du travail, s'apparente à un constat banal. Ce retournement général n'empêche pas Naville de poursuivre et de publier ses travaux empiriques et théoriques, dont la rigueur ne fait pas de doute (Naville, 1984), ni ses disciples d'approfondir ses conceptions sur la base d'une théorie marxienne du salariat (Rolle, 1986; Vincent, 1987).

\section{MUTATION DU RAPPORT SALARIAL, IDENTITÉ AU TRAVAIL ET SOCIÉTÉ DU RISQUE}

Au milieu des années 1980, la relance d'une approche similaire à celle de Naville vient de sociologues allemands formés par Adorno. Au vu de l'éloignement progressif de Touraine de la sociologie du travail, Horst Kern et Michael Schumann renouent avec le fil de la discussion des années 1960, au sujet de la rationalisation, de l'automation et de la transformation des activités de travail. Les deux auteurs constatent dès 1984 que les tendances de rationalisation capitaliste transforment de nouveau massivement le rapport au travail, à partir de grandes enquêtes réalisées en Allemagne (Kern et Schumann, 1984). En conclusion de leur analyse de l'automation et de l'informatisation dans les principaux secteurs industriels, ils énumèrent trois développements. Premièrement, ils constatent que les nouveaux dispositifs de travail diminuent forte- 
ment le besoin en main-d'œuvre peu qualifiée et augmentent la polyvalence des tâches, ce qui émousse la spécialisation tayloriste et rend pensable une plus grande autonomie pour les salariés qualifiés. Deuxièmement, cette évolution tend à marginaliser les travailleurs faiblement qualifiés, à les mettre au chômage et à les éloigner du marché de l'emploi. Par conséquent, la rationalisation engendre des inégalités et disparités nouvelles et fortes. Troisièmement, la distance se creuse, entre les structures de l'État social associées à des modalités de participation traditionnelles des ouvriers et employés et la modernisation managériale ou organisationnelle, qui est à l'œuvre dans les grandes entreprises.

Au même moment, Brandt (1991) examine le système de régulation allemand et souligne que cette évolution provoque une distanciation entre le personnel qualifié à l'intérieur des grandes entreprises d'une part, et le gros des salariés d'autre part, qui se retrouve dans le découplage entre la représentation des salariés à travers de puissants Betriebsräte (comités d'entreprise) dans l'industrie et des groupes moins protégés (ouvriers faiblement qualifiés, employés, femmes, travailleurs immigrés). Le résultat est l'émergence d'une importante fragmentation du salariat qui favorise la concurrence entre plusieurs groupes à travers des divisions sectorielles et sur le marché de l'emploi qui s'accompagnent d'attitudes défensives et corporatives (Brandt, 1991 : 210-229). Les réflexions de Brandt s'achèvent par le constat que les syndicats allemands ne sont plus en mesure de regrouper les intérêts divergents des salariés qualifiés et de la masse des petits métiers en voie de marginalisation qui cherchent de nouvelles formes de participation (Brandt, 1991: 380).

Ces recherches abordent et anticipent aussi des dispositifs du néo-management qui tendent vers une organisation flexible, partiellement autonome fondée sur la gestion par projet et l'auto-contrôle. Michael Schumann a montré que ces modes de gestion, loin de produire une adhésion spontanée de la part des salariés qualifiés, engendrent des lignes conflictuelles et des formes de résistance nouvelles. L'auteur insiste ainsi sur le décalage qui peut se faire jour entre la représentation ou le discours du néo-management (le nouvel esprit du capitalisme) et les pratiques des salariés (Schumann, 2003: 99). Au moment où la sociologie de l'action de Touraine ou encore la théorie de l'agir communicationnel d'Habermas délaissent l'analyse critique du travail, au bénéfice d'une observation culturelle de l'espace public, les observations empiriques et les conceptualisations critiques de la sociologie du travail signalent que l'ancien modèle de participation publique est en voie d'être défait (aujourd'hui, l'effet de cette dynamique apparaît en France dans l'abstention électorale majoritaire des ouvriers, employés et chômeurs). Friedmann avait compris l'extension du domaine de l'automatisation et de l'informatisation sur l'ensemble de la société et cherché à saisir la communication de masse qui en est l'une des extensions techniques, à travers des essais publiés à titre posthume où il aborde notamment le rôle de la télévision (Friedmann, 1979).

Si Kern, Schumann et Brandt arrivent à maintenir une analyse à la fois empirique et théorique du salariat en mutation, ils éprouvent cependant des difficultés méthodo- 
logiques à apprécier l'activité de travail sur les lieux de travail, au vu du caractère plutôt macrosociologique de leur approche. Horst Schumann tire un bilan de ses propres recherches en 2003, où il constate que les projets d'enrichissement du travail qu'il a accompagné, à l'instar des expérimentations des années 1990 chez Volkswagen, centrés sur la polyvalence, la rotation des tâches, le travail de groupe et les cercles de réflexion collective, se sont soldés par des échecs ou furent intégrés à une stratégie de modernisation managériale axée sur la productivité et la compétitivité qui a laissé peu de marge aux questionnements sur le rapport au travail (Schumann, 2003).

Dans le contexte global d'un glissement de l'analyse du travail vers l'organisation, plusieurs auteurs français ont cherché à relancer des observations du travail vécu et pratiqué sur les lieux de travail à travers une approche clinique. Le thème de l'humanisation du travail par l'enrichissement des tâches et des compétences s'est alors transformé en réflexions et observations cliniques sur l'identité au travail ou à la psychodynamique en milieu de travail. Ces approches, diverses, ont comme point commun de mobiliser des concepts et savoirs issus de la psychanalyse, appliqués au contexte des organisations (Sainsaulieu, 1984; Dejours, 1988; De Gaulejac, 2005). Le souci de l'humanisation du travail, cher à George Friedmann, a connu une relance à travers l'idée de la réalisation de soi, le "pouvoir d'être soi-même» (Sainsaulieu), et l'idée d'un sujet qui s'attribue lui-même le sens de l'action. Les enquêtes menées à partir de ces problématiques sont nombreuses et riches, elles vont des ateliers de réparation à la compréhension de l'univers d'IBM, en passant par le milieu hospitalier. Les chercheurs diversement influencés par l'œuvre de Pierre Naville ont remarqué l'absence d'une théorie du salariat et d'une critique globale des principes managériaux dans ces approches (Rolle, 1997; Vincent, 1987; Bouquin, 2008), mais ce n'est pas l'angle que nous retenons afin de progresser dans la compréhension du concept d'activité. Robert Castel, qui n'a pas développé de théorie du salariat, a ouvert la voie à une critique très circonstanciée de l'approche clinique au détour de la réédition de son livre La gestion des risques (Castel, 2011). Il constate que la culture psychologique, qui fait de «l'intensification du potentiel personnel et relationnel de l'individu l'objet de toutes les attentions», qui a été marginale au début des sciences humaines, est devenue un «modèle de bonne gouvernance des sociétés contemporaines»(Castel, 2011: 13). L'auteur salue les percées intellectuelles de Freud, qui a permis de mettre en relation les aspects de la normalité et de la pathologie, qui semblaient devoir s'opposer chez Durkheim, une problématique reprise sous la forme du couple souffrance-plaisir chez Dejours et d'autres. Castel souligne cependant que dans l'observation pratique, le travail sur soi perd son potentiel universel parce qu'il ne s'applique qu'à des cas individuels ou des groupes très limités (Castel, 2011: 164). Il est vrai que Freud a ébauché une théorie de la psychologie de masse qui a été prolongée par des sociologues de l'École de Francfort plus tard (Neumann, 2015), alors que dans les études sociologiques cliniques qui nous intéressent ici, l'analyse ne dépasse jamais un nombre assez limité de salariés, à l'échelle d'un atelier, d'un bureau ou d'un service. L'évolution globale de la société, de ses normes et dimensions pathologiques reste donc nécessairement à 
l'écart de ces études empiriques partielles. Castel inscrit cette limitation dans une situation historique où «les contraintes objectives de l'économie sont placées hors de l'atteinte des sujets, qui n'ont d'autre choix que de travailler l'espace de leur propre potentiel et de leurs relations» (Castel, 2011: 184). Ce dilemme a été désigné par le concept paradoxal de "l'action contrainte»(Courpasson, 2000). Chez Dejours, la difficulté d'inscrire les «attitudes défensives» (Freud parlait de réaction défensive) des salariés dans une compréhension sociopsychologique ou sociologique plus globale apparaît dans le fait qu'il se sent obligé de se référer au cadre plus large de la théorie de l'agir communicationnel d'Habermas (Dejours, 1999; Habermas, 1987). Pourtant, Habermas écarte explicitement le domaine de l'entreprise du champ d'application de sa théorie parce qu'il la considère comme une sphère privée qui est régie par le marché et l'action instrumentale (Neumann, 2015). Ce cadre théorique est donc singulièrement mal approprié pour réduire l'écart entre les situations de travail singulières et une théorie sociale plus globale. Friedmann a rencontré une difficulté similaire, lorsqu'il a tenté de séparer l'observation du travail du domaine du hors-travail et du temps libre, qui touche obligatoirement à tous les aspects de la vie sociale. Pour revenir au fil de l'argumentation de Castel, pareille éclipse de la dimension publique correspond au leitmotiv programmatique de la valorisation du «capital humain», qui sollicite l'accroissement des capacités individuelles dans l'unique optique de maximiser son propre potentiel au travail, dans le sens d'une «activation de l'individu» (Castel, 2011: 12). Cet appel à la maximisation des potentiels individuels et à la minimisation des pertes concorde avec une gestion des risques, où il s'agit d'évaluer en permanence les situations individuelles, depuis l'enfance jusqu'aux compétences des salariés. Nous ajoutons que cela s'étend jusqu'au risque des suicides au travail. C'est en ce sens précis que Castel comprend l'ouvrage La société du risque d'Ulrich Beck paru pour la première fois en 1986 (Beck, 2008).

Castel ne développe pas cet argument, mais il est possible qu'il ait davantage percé à jour le concept de "société du risque» que sa brève citation ne le laisse paraître. La flexibilité et l'activation du travail font partie de l'un des chapitres du livre de Beck qui décrit l'érosion des protections collectives. C'est précisément dans ce domaine que la notion de risque prend son sens, en tant que compromis social et sémantique, au cours d'une bataille qui opposa syndicats ouvriers et patronat depuis la seconde moitié du $19^{e}$ siècle sur la question de savoir si les ouvriers étaient individuellement responsables des accidents de travail ou si c'était le patronat en tant qu'employeur. La notion de risque a déplacé l'enjeu vers un registre assurantiel qui fait abstraction des situations singulières par le calcul et la socialisation des coûts. L'origine du mot se trouve dans la garantie contre le péril, c'est le prix pour éviter un danger qui donne lieu au crédit et à l'assurance commerciale (Thiveaud, 1996). Par conséquent, la logique du risque incarne une conception entièrement basée sur l'aspect macroéconomique de l'organisation, au détriment de l'activité et des situations de travail vécues. Dans un entretien tardif, Beck a d'ailleurs dénoncé le développement concurrentiel que cela autorise et qui s'apparente selon lui à une forme de darwinisme social (Beck, 5/2/2010). 
En Allemagne, on constate une évolution théorique qui va du primat de la Industriesoziologie (sociologie des relations industrielles influencée par Friedmann et Naville) des années 1960 et 1970, vers la Betriebssoziologie (la sociologie de l'entreprise dont le terme allemand remonte à Weber) pour arriver à la Organisationssoziologie ou plus récemment la Wirtschaftssoziologie (la sociologie de l'économie). Il serait sans doute possible de retracer une telle évolution du champ sociologique à travers la succession des approches qui ont prévalu en Grande-Bretagne à différentes époques, en partant des industrial relations, en passant par le labour process pour arriver aux labour market studies et les expertises sur le new public management, mais cela déborde notre champ de compétences ${ }^{1}$. Le long cycle des recherches sociologiques qui a conduit à une primauté du discours de l'organisation et de la vision macrosociologique fait que les enquêtes de terrain locales ou thématiques ont été largement englobées dans cette vue d'ensemble, sur le mode scientifique d'une validation empirique, y compris lorsque l'exception vient confirmer la règle. Dans ce cadre, l'importante monographie sur la condition ouvrière aux usines Sochaux-Montbéliard de Beaud et Pialoux (1999), suivie d'une analyse complémentaire sur le même site présenté par Durand et Hatzfeld (2002), n’a eu pratiquement aucun effet sur l'orientation générale de la sociologie du travail. Il est vrai que leurs auteurs entendaient appliquer des théories sociologiques bien établies, l'ethnographie ouvrière et la théorie de la reproduction scolaire introduite par Pierre Bourdieu pour Beaud et Pialoux; une lecture marxiste assez traditionnelle du conflit capital-travail pour les seconds. Les enquêtes d'ethnographie ouvrière qui se réferent à Bourdieu, comme celle de Beaud et Pialoux, celle d'Olivier Schwartz sur les chômeurs du département du Nord (Schwartz, 1986) ou encore celle de Florence Weber sur une petite commune industrielle dans une région rurale (Weber, 1989), apportent toutes des connaissances précieuses sur des milieux de travail, mais ne prétendent pas apporter une conceptualisation théorique globale du travail. En ce sens, elles se soustraient au leitmotiv de l'organisation sans le contredire explicitement sur le terrain théorique qui le fonde.

La sociologie de Bourdieu elle-même ne recourt pas à une théorie générale du travail. Bien que Marx fasse partie des principaux auteurs mobilisés dans les écrits qui portent sur la transformation de la société algérienne (Bourdieu, 1977), l'auteur ne mobilise pas la théorie du salariat d'inspiration marxienne. Castel, quant à lui, la refuse explicitement dans sa caractérisation historique du prolétariat et de ses transformations (Castel, 1995: 329). Au cours de La misère du monde (Bourdieu, 1993), qui expose de nombreuses situations de travail, ne se trouve aucun concept sociologique particulier du travail ni dans les écrits théoriques de Bourdieu (Neumann, 2010: 121-134). Dans La Distinction, Bourdieu (1979) fournit une clé qui permet de saisir cette orientation conceptuelle. Il y affirme que les ouvriers, employés et autres catégories subalternes ne sauraient agir par eux-mêmes, selon leurs propres conceptions, à partir de l'expérience

1. Nous remercions Bruno Lefèbvre, Michel Lallement et Steve Jeffries de ces remarques et de leur discussion ad hoc de notre intervention lors de la journée d'étude du LISE-CNRS Les sociologies du travail en Europe, CNAM, Paris (5/5/2015). 
du travail, mais se trouveraient réduits à exécuter ce que la bourgeoisie leur dit de faire (Bourdieu, 1979: 538). Aussi, le concept central de capital symbolique ne trouve pas d'équivalent dans une notion touchant au travail, qui aurait pu s'appeler le travail symbolique, par exemple. Si l'approche bourdieusienne s'oppose explicitement à la sociologie de l'action de Touraine et à la théorie de l'agir communicationnel d'Habermas qui lui correspond, elle ne répond pas directement à la position philosophique qui inspire leurs interprétations du travail, à savoir la conceptualisation que propose Hannah Arendt (Touraine, 1999; Habermas, 1981). À la suite d'une revue des anciens philosophes grecs, la philosophe définit le travail comme un objet apolitique qui appartiendrait au domaine privé et au faire (tun), alors que la délibération citoyenne et la participation à l'espace public expriment l'agir (handeln), selon elle (Arendt, 1961). Sa lecture repose sur une opposition catégorique entre l'oikos (domaine privé) et l'agora (espace public) dont le caractère dichotomique a été interrogé (Negt, 2001). La conceptualisation arendtienne a aussi été développée, de manière fidèle, dans le débat français sur la fin du travail ou la souffrance au travail (Meda, 1998; Dejours, 1999). En théorie, l'entreprise n'est pas concernée par l'agir, mais le niveau analytique de l'organisation globale de la société semble y correspondre. Sur le plan des enquêtes empiriques sur le travail, l'on constate ainsi un déplacement du centre de gravité similaire à celui qui s'est produit sur le plan théorique, depuis les années 1960. Les grandes enquêtes ouvrières sortent du centre de l'attention pour laisser une place plus grande aux principaux acteurs de l'organisation, les cadres, leurs discours et pratiques (Boltanski, 1980; Boltanski et Chiapello, 1999; Cousin, 2008; Dujarier, 2015). Dans la même période qui a vu émerger la conception de la fin du travail, le développement massif de contrats de travail précaires a été associé à la précarité prolétaire des petits boulots et au chômage (Castel, 1995), alors que la précarité contractuelle touche tous les métiers, y compris des qualifications élevées dans la recherche ou la culture (Cingolani, 2014). Il s'agirait donc de réintégrer cet aspect dans une sociologie plus générale.

La séparation stricte des catégories du faire et de l'agir, d'inspiration arendtienne, a aussi des répercussions sur la perception de la division sexuée du travail et la sociologie féministe. Nancy Fraser a regretté la séparation théorique et pratique, entre d'une part un féminisme centré sur la construction des identités sexuelles, tourné vers un paradigme culturel, et d'autre part un féminisme sociologique qui analyse les rapports de complémentarité entre le travail domestique, le travail salarié et la participation publique (Fraser, 2012). Pareille complémentarité a été esquissée par Negt et Kluge, qui ont associé le travail vivant, opposé au capital, et le travail domestique (Hausarbeit), dès les années 1980 (Negt et Kluge, 1981), dans un dialogue direct avec des sociologues féministes (Axeli-Knapp et Becker-Schmidt, 2000).

Dans la constellation d'une suprématie théorique manifeste des paradigmes centrés sur l'organisation et l'action, des sociologies alternatives ont continué à se développer, dont les auteurs se réfèrent aux écrits fondateurs de Friedmann (Lallemant), de Naville (Rolle) ou parfois de Theodor W. Adorno (Kern, Schumann et Negt). Les argumentations marxistes ont continué à jouer un rôle critique remarquable (Linhart, 
1991; Friot, 1998; Bouquin, 2006) sans pour autant parvenir à changer les termes du débat général, qui est longtemps resté dominé par le lexique du risque, de la société postindustrielle, flexible, informationnelle, communicationnelle ou cognitive. Il nous semble que l'expérience de la crise économique ouverte en 2008 et le constat des limites des conceptions de l'activation aient favorisé une relance des approches critiques anciennes ou novatrices (Dörre, Rosa et Lessenich, 2009; Neumann, 2010; Mercure et Vultur, 2010; Cingolani, 2012; Sauer et Wittke, 2012).

Depuis 2008, l'année qui marque la manifestation de la crise économique mondiale, la règle elle-même apparaît comme problématique, à travers un fort débat public au sujet de la régulation économique et financière (à titre d'exemple: Piketty, 2013) qui thématise les inégalités sociales et le droit du travail. Même si ce tournant du débat économique se place encore sur un plan d'abstraction qui ne comprend pas les situations de travail en tant que telles, comme l'a pratiqué l'École de la Régulation, il a néanmoins provoqué une discussion sociologique sur le sens de la règle établie, et sur les critères de jugement pertinents. La règle elle-même est entrée en crise et les sciences sociales s'en font l'écho. Cette interrogation ne s'arrête nullement au rôle des agences de notation, mais intervient désormais dans la sociologie elle-même. Ainsi, plusieurs sociologues allemands se sont efforcés de réanimer l'héritage critique de la discipline afin de mettre en question le sens de l'organisation. Dörre, Rosa et Lessenich soulignent les limites intrinsèques du modèle d'organisation concurrentiel et assurantiel qui est porté par les catégories de risque, de gestion et de rationalisation. Reprenant les termes du débat des années 1960 et 1970, qui avaient été éclipsés en grande partie par le tournant vers l'organisation, notamment l'automatisation sociale et la question de l'accélération temporelle, ces auteurs s'emploient à montrer que la déconnexion entre temps de travail et temps travaillé a abouti à une accélération des activités qui commencent à peser sur les conditions de travail et de vie, par le stress continu, l'invasion de la sphère intime par le temps de l'entreprise et le temps entrepreneurial, provoquant des souffrances et symptômes psychiques en masse qui peuvent être mesurés sur les lieux de production et par des moyens statistiques. Les travaux de Hartmut Rosa (2013) à ce sujet ont connu une réception significative, tandis que le livre avant-coureur de Peter Glotz (1999) qui avait abordé exactement la même problématique n’a jamais été traduit ni discuté, ce qui tend à accréditer notre thèse d'une remise en question récente du discours de l'organisation. Un théoricien et sociologue de premier plan de ce discours est venu confirmer ce changement à son tour, il s'agit de Wolfgang Streeck. Dans les années 1990, cet auteur avait conceptualisé la nécessaire soumission du travail et du syndicalisme aux normes concurrentielles globales, selon le modèle du «corporatisme concurrentiel» et national qu'il préconisa alors (Streeck, 1999). Il s'agit d'une alliance des organisations de masse (partis, syndicats, organisations patronales) dans l'objectif d'augmenter la compétitivité nationale au détriment des salaires, des conditions et des statuts de travail, revus à la baisse. Récemment, le même Streeck a reconnu le relatif échec de ce modèle, qui n'a fait que retarder les problèmes, d'où le titre de son livre, Du temps acheté (Streeck, 2014), où il vient rejoindre nombre d'arguments de Dörre, 
Rosa et Lessenich. Dans son introduction, Streeck va jusqu'à s'exclamer qu'Adorno avait raison au sujet de ses thèses critiques envers le capitalisme tardif en 1968 (Adorno, 2012), sa seule erreur aurait été d'avoir eu raison trop tôt. D'autres sociologues du travail ont ouvert une discussion sur les apports théoriques des critiques du capitalisme et ses normes de gestion actuelles (Sauer et Wittke, 2012), alors que le concept de travail fait son entrée dans l'encyclopédie des théories critiques (Demirovic, 2016).

\section{L'ACTIVITÉ CONTRE L'ACTIVATION ?}

Le commentaire de Castel au sujet de «l'activation de l'individu» dans une stratégie globale de valorisation du capital humain peut s'appliquer parfaitement au programme Agenda 2010 de l'Union européenne que les chefs d'État ont arrêté en 2000 lors du sommet de Lisbonne (UE, 2000). Le principe d'activation est omniprésent dans la déclaration de Lisbonne, qui entend faire de l'Europe «l'économie du savoir»» la plus dynamique et compétitive du monde, afin d'atteindre le plein emploi dès 2010. Il s'agit en quelque sorte du point culminant d'une évolution du travail pratiqué vers l'organisation abstraite que nous souhaitons mettre en relief. Le leitmotiv de l'économie du savoir implique l'extension des services, le développement des compétences «postindustrielles» et une plus grande mobilité territoriale. Ces objectifs furent ensuite déclinés à travers la réforme des agences pour l'emploi (activer les chômeurs et les inciter à se former, à se reconvertir ou à devenir mobiles), du droit du travail et de l'éducation (la qualification et les métiers perdent leur importance au vu d'une formation tout au long de la vie). En France, le revenu social d'activité (RSA) porte la trace de ce dispositif global.

À notre sens, le concept sociologique d'activité et le principe gestionnaire de l'activation peuvent cependant se révéler antinomiques. Le premier évoque le travail pratiqué, tandis que le second, une gestion quantitative, abstraite et désincarnée. Sur le plan sociologique, le principe de l'activation s'appuya sur les thèses de Beck (2001), de Giddens (1994) et de Streeck (1999), toutes très éloignées de l'observation des situations de travail. Ce type de conception est encore radicalisé dans l'essai théorique et économique de Yann Moulier-Boutang, Le capitalisme cognitif, qui imagine une économie entièrement fondée sur le «travail immatériel» (Moulier-Boutang, 2007). Dans ces conceptions, la société du savoir et de l'information semble résorber le travail, tandis que les représentations administratives et la quantification macroéconomique priment sur la qualité. Pourtant, les travaux empiriques sur le travail numérique (digital labor) signalent la persistance et l'importance des activités corporelles et physiques des tâches supposément immatérielles (Scholz, 2013). Internet lui-même présente des traits analogues à une usine industrielle (en termes de standardisation et de gestion en masse), sans oublier le fait que l'activité cérébrale implique un effort physiologique qui peut atteindre une dépense d'énergie de sportif de haut niveau. Les activités industrielles, anciennes ou nouvelles, sont désormais imbriquées dans une gestion informatique de la production, de la logistique et de la distribution, à l'image de la mécatronique ou des réseaux d'énergie intelligents. Le ministère allemand de l'Économie nomme ce 
phénomène Industrie 4.0., tandis que le syndicat de la métallurgie aborde le travail 4.0. (Wetzel, 2015). Conceptuellement, Naville avait anticipé ce processus global par l'idée de l'automatisme social (Naville, 1957b).

Le dispositif de Lisbonne de l'UE a institutionnalisé le discours de l'activation, de l'organisation, de la rationalisation et du risque. Il a inauguré une méthode qui cherche à unifier les particularités sociales, nationales, historiques des pays membres et de leurs relations industrielles, en décidant de traiter tous les problèmes thématiques et les conflits intergouvernementaux selon un schéma managérial connu de la sociologie des organisations, en fixant par avance des procédures et des étapes dans le cadre d'un programme de dix ans, l'Agenda 2010. Il s'agit d'une sorte de management par projet inspiré des grandes entreprises, appliqué à la construction européenne, selon la MOC, la «méthode ouverte de coordination» (Bruno, 2008). La MOC fonctionne selon le principe de gestion du «benchmarking» international, consistant à identifier les «meilleures pratiques » et les points faibles des dispositifs nationaux respectifs, à partir d'une grille d'analyse préétablie qui mesure les performances et contre-performances de manière quantitative. Les défauts constatés à travers la MOC doivent être améliorés par des plans d'action chiffrés comportant des délais précis. Pareille logique se décline ensuite sur le plan des ministères, des régions, etc. Les chiffres ainsi obtenus sont désormais traduits en coûts à travers un logiciel économique, le SCM (Standard Cost Model) que nous avons pu expérimenter (Neumann, 2011). Le SCM prétend calculer toutes sortes de politiques publiques en termes de coût économique, son ambition est donc de quantifier toute action en termes monétaires, sans égard aux particularités historiques, sociales, régionales ou de genre. L’activité réelle des salariés ne joue ici aucun rôle. Le général écrase le particulier. En pratique, la méthode prévoit d'interroger des entrepreneurs, administratifs ou décideurs, afin d'obtenir de leur part une évaluation ad hoc des coûts, charges et efforts de travail que représentent des actes de routine (ou encore des changements dans cette routine). Sitôt calculée, l'évaluation plutôt intuitive des indicateurs, basée sur une expérience personnelle, se présente sous la forme de faits incontestables qui servent de référence. De manière analogue à la production de sondages d'opinion, la méthode d'observation, d'expertise et d'évaluation unique de la Commission européenne organise une représentation mathématique abstraite dont le principe même gomme les expériences et activités concrètes.

Du point de vue des institutions européennes, il ne s'agit pas d'imposer un modèle unique d'organisation du marché de l'emploi via une directive cadre, rendue impossible par l'absence de droit social européen, mais d'inciter les gouvernements des pays membres à prendre des mesures concrètes favorables à la flexibilité et de comparer les résultats à travers un benchmarking permanent. Le benchmarking se réfere aux calculs obtenus grâce au SCM. La Commission européenne demande ici aux gouvernements d'orienter leurs réformes nationales autour des lignes directrices de la flexicurité, d'en rendre compte annuellement et d'en justifier le bilan en 2011, à la fin de l'Agenda 2010. Son propre bilan souligne les progrès dans l'intégration des programmes nationaux dans les lignes directrices de la Commission, qui furent parfois déclinés jusqu'aux 
politiques régionales, et le fort impact pratique de cette stratégie dans les pays membres, malgré des variations significatives. Au moment où le taux de chômage au sein de l'UE a atteint le record de $11 \%$, le document de bilan affirme que la stratégie a eu un «impact très positif sur l'emploi », dynamique qui n'aurait été entravée que par la crise économique en 2009. Tous les autres objectifs chiffrés arrêtés au début de l'Agenda ont connu le même échec, de la résorption des inégalités entre travailleurs faiblement et fortement qualifiés, de la diminution des inégalités de genre, entre jeunes et vieux, entre régions européennes, etc. Désormais, il s'agit de sortir de ce cadre normatif qui correspond aussi à une certaine conception sociologique qui se bute à ses propres limites, afin de permettre une compréhension qualitative du travail que la sociologie du travail avait inaugurée dans les années 1960.

Le concept d'activité s'inscrit dans le renouveau de la discussion sociologique qui prospère au milieu de la crise du dispositif organisationnel établi. En apparence, il s'agit d'une notion empirique, susceptible de nommer les innombrables situations et pratiques de travail qui ne sont pas représentées par le discours de l'organisation et de l'activation. En ce sens, Alexandra Bidet parle du «vrai boulot», à partir d'enquêtes qui précisent l'investissement personnel dans le travail (Bidet, 2011). L'approche, qui favorise une mise en évidence des sujets en action, rappelle celle qui avait permis à Friedmann de dépasser les lacunes doctrinaires de Durkheim, grâce au recours à des observations empiriques, en l'occurrence les activités qui dépendant du trafic téléphonique (qui illustre l'extension du domaine de l'automation observée dès les années 1950). Cette mise à jour, à partir d'un milieu de travail contemporain, permet de dépasser le schéma implicite du travail artisanal qui est encore présent chez Friedmann, et qui a ressurgi chez Touraine sous la forme de l'idéalisation philosophique de l'œuvre à l'encontre du travail qui dérive de l'argumentation d'Arendt. La notion d'activité permet in fine de reposer la question théoriquement fondamentale du rapport entre le faire et l'agir. Si Friedmann s'est focalisé sur le faire (artisanal), la sociologie de l'action et la théorie de l'agir communicationnel que nous avons déjà abordées se réfèrent exclusivement à l'agir arendtien. Comment passer du faire à l'agir sur le plan théorique? Friedmann était conscient de la difficulté à saisir la société dans sa globalité à partir des observations partielles qu'il avait réalisées sur le travail. Comment saisir la dynamique de la société sur la seule base de tels éléments, puisqu'il avait souligné — à l'encontre de Durkheim — l'importance de la solidarité ouvrière en dehors de l'entreprise, de la coopération sociale et avait laissé en suspens la définition du hors-travail? Jean-Marie Vincent (1987) a bien tenté d'ébaucher l'association du faire et de l'agir, à la suite de Naville, avec un certain éclat intellectuel, sans jamais solliciter des observations empiriques denses, ce qui a considérablement limité la portée de son propos. Il est vrai que le marxisme structuraliste avait neutralisé cette question, en postulant une unité entre travail et politique, à travers l'idée d'une constitution automatique du conflit de classe. S'agissant de la proposition de Bidet, Bernard Francq constate que l'effort de passer de la compréhension d'un milieu de travail circonscrit à une théorie sociologique ou anthropologique générale (à l'aide d'auteurs comme Luc Boltanski ou 
Bruno Latour), qui permettrait de déployer le «grand récit du travail», est louable et représente un énorme défi (Francq, 2013). Pour l'instant, l'écart reste grand, entre le travail en actes et la conceptualisation du travail. Pour ne retenir qu'un seul exemple, Luc Boltanski et Ève Chiapello (1999) ne prennent pas en considération les expériences pratiques du travail, car les deux auteurs ont fait le choix méthodologique délibéré de construire leur analyse à partir des productions discursives et textuelles du management pour saisir le nouvel esprit du capitalisme.

La notion d'activité nous semble propice pour prolonger le débat ouvert par la crise du discours de l'organisation et de l'activation. Débat qui incite la sociologie du travail à se souvenir de la vaste culture philosophique et conceptuelle qui a animé ses fondateurs, de prendre au sérieux l'ampleur de son programme empirique, humaniste et critique, afin de comprendre les deux dimensions du faire et de l'agir, et de se hisser à la hauteur de la grande transformation qui travaille les sociétés contemporaines.

\section{CONCLUSION : CONCEPTUALISER LE TRAVAIL ET L'ACTION}

Le développement théorique que nous avons cherché à retracer dans ses grandes lignes peut se lire comme celui d'une sociologie du travail qui s'est muée, dans son versant le plus visible, en une sociologie qui a perdu la notion du travail. Cette dissolution conceptuelle du travail a emprunté plusieurs voies, parfois sinueuses, souvent convergentes qui ont toutes abouti à l'invisibilité du travail comme activité réelle. L'une des voies a suivi la piste philosophique d'Hannah Arendt, qui a voulu troquer le concept supposément acritique de travail contre celui d'action, opposant ainsi le faire et l'agir. Touraine et Habermas ont explicitement choisi cette orientation. Ils ont lancé un mouvement qui a remplacé le travail par la culture, puis la communication. Dans la filiation tourainienne, Manuel Castells a déployé une sociologie de la communication où le terme de travail n'affleure jamais, et son index alphabétique ne le nomme pas, au milieu d'entrées comme la tour Eiffel, Texaco ou Transparency International (Castells, 2013). Dans la tradition habermassienne, Axel Honneth a voulu écarter la sociologie du travail comme une science purement gestionnaire (Honneth, 1980: 185), avant de se raviser récemment dans son livre Le droit de la liberté (Honneth, 2012). Dans l'une des grandes parties, il tente de définir le marché de l'emploi comme «l'objet empirique» d'une analyse des conditions de la liberté sociale, mais il ne mobilise en réalité aucune étude empirique dans ce domaine, car il entend l'empirisme comme pure approche philosophique, dans la tradition anglaise. En revanche, il s'appuie sur les thèses initiales de Durkheim concernant la division sociale du travail et de la solidarité organique, auteur qu'il considère comme le père fondateur de la théorie politique d'Habermas, bien que la sociologie du travail moderne ait compris les limites de ce modèle sociologique, depuis Friedmann.

Conjointement, les théories de l'information ont cru que l'activité de travail allait se dissoudre dans le flux numérique, dans le cadre d'une société postmatérielle, alors que les spécialistes du travail numérique montrent, avec Scholz, que celui-ci présente précisément tous les aspects du travail (Scholz, 2013). Pendant ce temps, 
le travail salarié, industriel et traditionnel perdure sous des formes plus ou moins adaptées au contexte mondial et technique, ce que Scholz ne prend pas directement en considération.

Les théoriciens de la société du savoir et du capitalisme cognitif ont cru à leur tour que le travail allait se fondre dans la masse des activités sociales, alors que la crise économique globale a rappelé la matérialité des questions de revenu, de rémunération, de chômage et de qualification.

Enfin, l'organisation collective du travail aurait été abolie par une gestion des risques, à travers la société du risque, avant que les auteurs majeurs de cette approche ne se ravisent, à la manière de Beck et Streeck.

S’il fallait trouver une racine commune à toutes ces approches, qui sont entrées en crise ces dernières années, il serait possible de la désigner dans une lecture plutôt conservatrice de la Phénoménologie de l'esprit d'Hegel (1803), plus précisément de son chapitre «Le maître et le valet» (Herr und Knecht). Le philosophe allemand écrit alors sous le choc de la Révolution française, donc dans un moment de crise où les critères de jugement et la pertinence des concepts sont interrogés ouvertement. Dans ce texte, il déploie tout l'arsenal conceptuel que la pensée du travail a exploré ultérieurement, en commençant par l'existence (Dasein) en passant par le faire (Tun), le travail (Arbeit), la reconnaissance (Anerkennung), la conscience (Bewusstsein), pour finir sur une méditation au sujet de la résistance, sous sa forme de subjectivité rebelle (Eigensinn), qu'il définit comme une «liberté qui reste encore à l'intérieur de la servitude». Hegel suggère une succession d'étapes conceptuelles et historiques qui interagissent et sont donc liées de manière dialectique, contrairement à l'opposition schématique, entre faire et agir, qui fut introduite par Touraine et Habermas à la suite d'Arendt. La discussion sociologique souffre aujourd'hui de la séparation artificielle des concepts en débat. Si le terme même de l'existence est associé à Heidegger ou Sartre (malgré le désaccord violent qu'Heidegger a exprimé contre l'humanisme existentialiste), le travail se trouve accolé à la pensée de Marx, élève d'Hegel, qu'Heidegger a désigné comme son adversaire principal. La reconnaissance se trouve en titre d'un livre d'Honneth (1998), et dans le débat philosophique français sur la souffrance et la reconnaissance, qui manifeste une opposition au marxisme hégélien. Enfin, le Eigensinn est le leitmotiv d'un livre de Negt et Kluge, tous deux formés par Adorno, et qui ne fut jamais traduit vers le français (Negt et Kluge, 1981).

Adorno avait mis en garde l'ensemble des théoriciens et sociologues qui participaient à la discussion sur le travail, au sujet d'un penchant de la philosophie qui pense pouvoir résorber le travail dans le jeu conceptuel et ainsi dans l'esprit du temps, dans ses Trois études sur Hegel (Adorno, 2003). Il y souligne que le travail et les formes de résistance, conservatrices, identitaires ou critiques, ne disparaîtront jamais, mais vont simplement continuer leur vie en dehors du cadre théorique des sciences sociales si l'on choisit de les exclure. Negt a pour sa part insisté sur l'artificialité d'une séparation catégorique du travail et de l'action, et il a constaté l'impossibilité de concevoir un paradigme de la communication qui serait privé du travail comme source et aiguillon 
critique (Negt, 2007). Comme nous avons signalé l'ampleur des cultures philosophiques et épistémologiques qui sont intervenues lors de la fondation de la sociologie du travail, cela donne une idée du défi qui reste à relever à présent.

Nous ne prétendons pas résoudre ces problèmes paradigmatiques qui engagent un débat collectif très ample sur les perspectives des sciences sociales. En conclusion du présent article, nous soumettons une simple piste au débat qui part de notre constat général. Pendant près d'un demi-siècle, la tendance la plus forte était celle d'une marginalisation théorique du travail, parfois jusqu'à sa dissolution conceptuelle dans le langage de l'action, de l'organisation, du management, du risque, de l'activation. Ce mouvement fut assorti d'une minorisation de l'activité pratique, malgré d'excellentes enquêtes qui ont exposé le sens et l'importance de ce vécu, qui fut souvent ramené à un vécu individuel ou de groupes particuliers, sans lien avec la sociologie générale. L'idée d'un travail sans qualités s'est ainsi répandue à travers des critères quantitatifs, sinon macro-économiques, qui ne considèrent que le nombre. Pareille représentation se prolonge encore à travers le numérique, comme le montre la thèse du capitalisme cognitif.

L'expérience massive qui se produit à travers la crise globale ouverte en 2008, parmi les salariés stables, fonctionnaires, précaires, travailleurs indépendants ou demandeurs d'emploi, nous semble désormais provoquer un possible retournement conceptuel, qui placerait l'expérience partagée du travail à la base de la reconstruction du bien commun. Pour revenir à la philosophie d'Hegel, il peut se produire une transformation dialectique de la quantité en qualité. La discussion, en grand nombre, d'expériences de travail singulières mais comparables engage un débat public ample, comme cela peut notamment s'observer en Europe du Sud ou encore à la faveur du mouvement social opposé à la loi El Khomri en France, au printemps 2016, loi qui s'est présentée comme une application de la stratégie de l'activation dès son exposé des motifs. Ce débat semble ouvrir un espace aux chercheures et chercheurs dont les travaux ne sont pas centrés sur le paradigme de l'organisation et de la communication, mais plutôt sur le sens du travail comme une forme d'agir.

Depuis un demi-siècle, les survivances ou nouvelles formes du travail artisanal, les résistances du travail vivant à l'encontre de la soumission réelle du salariat, les observations ethnographiques sur les milieux de travail particuliers, et les dissonances entre sphère privée et publique ont interrogé les théories sociologiques. Du point de vue conceptuel, la philosophie adornienne (Adorno, 2003) indique que le mouvement de totalisation théorique dominant, qui tend à dissoudre le travail et l'expérience singulière dans le concept général, bute nécessairement sur des résistances, dans un retour à la racine du travail et de l'expérience. Il s'agirait de saisir ce mouvement d'aller et de retour sur le plan de l'interprétation sociologique.

\section{RÉSUMÉ}

La discussion qui se déploie actuellement autour du concept d'activité nous invite à interroger le développement théorique de la sociologie du travail, depuis les travaux fondateurs des années 
1950 et 60, à travers plusieurs tournants paradigmatiques successifs, jusqu'aux enjeux présents. La lecture théorique que nous poursuivons est donc celle d'un détachement progressif du travail pratiqué, qui aboutit à une focalisation sur l'organisation, du moins dans les courants les plus visibles de l'espace académique et public. Dans ce cadre, nous comprenons la notion d'activité à la fois comme un effort de recentrer le regard sociologique sur le travail en actes qui est exercé et vécu par les salariés, et comme une conception alternative à la vision d'un travail sans qualité (sous sa forme prétendument interchangeable, cognitive ou numérique).

Mots clé: activation, automatisme social, agir communicationnel, conscience ouvrière, travail sans qualités

\section{ABSTRACT}

The ongoing discussion around the concept of activity is an invitation to question the theoretical development of sociology of work, from the foundational works of the 1950 s and 1960s, through several successive paradigmatic shifts, up to today's issues. The theoretical reading we are pursuing is therefore that of a gradual turning away from practised work that has ended up focusing instead on the organization, at least among the most visible currents of academic and public space. Within this framework, we consider the notion of activity as an effort to refocus the sociological perspective on work in acts that are carried out and experienced by wage earners, and at the same time as an alternative to the notion of unspecialized work (under its allegedly interchangeable, cognitive or digital form).

Key words: activation, social automatism, communicative action, workers' consciousness, unspecialized work

\section{RESUMEN}

La discusión que se lleva a cabo actualmente alrededor del concepto de actividad nos invita a interrogar el desarrollo teórico de la sociología del trabajo, desde los trabajos fundadores de los años 1950 y 60, a través de varios pivotes paradigmáticos sucesivos, hasta los más actuales desafíos. La lectura teórica que desarrollamos es entonces la del desprendimiento progresivo del trabajo practicado, que lleva a una focalización en la organización, al menos en las corrientes más visibles del espacio académico y público. En este contexto, comprendemos la noción de actividad como un esfuerzo por centrar la mirada sociológica en el trabajo en actos ejercidos y vividos por los asalariados y, a la vez, como una concepción alternativa de la visión de un trabajo sin cualidad (bajo su forma pretendidamente intercambiable, cognitiva o numérica).

Palabras clave: activación, automatismo social, actuar comunicacional, conciencia obrera, trabajos sin cualidades

\section{BIBLIOGRAPHIE}

Adorno, Th. W. (2012), Société, Paris, Payot.

Adorno, Th. W. (2003), Trois études sur Hegel, Paris, Payot.

Arendt, H. (1961), Condition de l'homme moderne. Vita Activa, Paris, Calman-Lévy.

Axelli-Knapp, G. et Becker-Schmidt, R. (2000), Feministische Theorien, Hambourg, Junius.

Beaud, S. et Pialoux, M. (1999), Retour sur la condition ouvrière, Paris, Fayard.

BECK, Ulrich (2001), La société du risque, Paris, Aubier.

BECK, Ulrich (1987), Risikogesellschaft, Francfort-sur-le-Main, Suhrkamp. 
Bidet, Alexandra (2011), L'engagement dans le travail. Qu'est-ce que le vrai boulot?, Paris, Presses universitaires de France.

Boltanski, L. (1980), Les cadres, Paris, Les Éditions de Minuit.

Boltanski, L. et È Chiapello (2009), Le nouvel esprit du capitalisme, Paris, Gallimard.

Bourdieu, P. (1977), Algérie 60, Paris, Éditions de Minuit.

Bourdieu, P. (1979), La Distinction, Paris, Seuil.

Bourdieu, P. (dir.) (1993), La misère du monde, Paris, Seuil.

BouQuin, Stephen (2006) «Domination au travail, ou domination du travail abstrait?», in Variations, ${ }^{\circ} 8$, éditions Parangon, Lyon, p. 76-89.

BRUno, Isabelle (2006), Déchiffrer l'Europe compétitive: Étude du benchmarking comme technique de coordination intergouvernementale dans le cadre de Lisbonne, thèse de doctorat.

Castel, R. (1981), La gestion des risques, Paris, Les Éditions de Minuit.

Castel, R. (1995), Les métamorphoses de la question sociale, Paris, Fayard.

Castells, M. (2013), Communication et pouvoir, Paris, Éditions de la Maison des sciences de l'homme.

Cingolani, Patrick (dir.) (2012), Un travail sans limites?, Paris, Érès.

Cousin, O. (2008), Les cadres à l'épreuve du travail, Presses universitaires de Rennes.

Crozier, M. (1963), Le phénomène bureaucratique, Paris, Seuil.

Crozier, M. (1971), La société bloquée, Paris, Seuil.

Crozier, M. et Friedberg, E. (1981), L'acteur et le système, Paris, Seuil.

Commission européenne (2010), Lisbon Strategy Evaluation document, Bruxelles.

Courpasson, D. (2000), L'action contrainte, Paris, PUF.

Dejours, Ch. (1988), Plaisir et souffrance dans le travail, édition de l'AOCIP.

Dejours, Ch. (2000), Souffrance en France, Paris, Seuil.

De Gaulejac, V. (2005), La société malade de la gestion. Idéologie gestionnaire, pouvoir managérial et harcèlement social, Paris, Seuil.

Dörre, K., Rosa, H. et Lessenich, S. (2009), Soziologie — Kapitalismus — Kritik, Berlin, Suhrkamp.

Dujarier, M. (2015), Le management désincarné, Paris, La Découverte.

Durand, J.-P. et Hatzfeld, N. (2002), La chaîne et le réseau, Lausanne, Page 2. Durkheim, E. (1990), Leçons de sociologie (1890-1900), Paris, PUF.

Durkheim, E. (2007), De la division sociale du travail social, Paris, PUF.

Durkheım, E. (1928), Le socialisme, Montréal, UQÀM (édition numérique): http://classiques.uqac.ca/ classiques/Durkheim_emile/le_socialisme/le_socialisme.pdf

FrancQ, B. (2013), «Recension d'Alexandra Bidet, L'engagement dans le travail, in La nouvelle revue du travail, 2/2013.

Fraser, N. (2012), Le féminisme en mouvements, Paris, La Découverte.

Friedmann, G. (1964), Le travail en miettes, Paris, Gallimard.

FriedmanN, G. (1966), Septétudes sur l'homme et la technique. Le pourquoi et le pour quoi de notre civilisation technicienne, Paris, Gonthier.

Friedmann, G. (1979), Les merveilleux instruments. Essais sur la communication de masse, Paris, DenoëlGonthier.

Friedmann, G. et Naville, P. (dir.) (1962) Traité de sociologie du travail (2 tomes), Paris, Armand Collin. Friot, B. (1998), Puissances du salariat, Paris, La Dispute.

Frisby, D. (1972), «The Popper-Adorno Controversy. The Methodological Dispute in German Sociology, in Philosophy of the Social Sciences, $\mathrm{n}^{\circ}$ 2, Sage, p. 105-119.

Giddens, A. (1994), Beyond Right and Left, Stanford University Press.

Glotz, P. (1999), Die beschleunigte Gesellschaft. Kulturkämpfe im digitalen Kapitalismus, Munich, Kindler. Habermas, J. (1981), Theorie des kommunikativen Handelns (2 tomes), Francfort-sur-le-Main, Suhrkamp.

Habermas, J. (1984), L'agir communicationnel, Paris, Fayard.

Hegel, G. W. F. (1807), Phänemenologie des Geistes, Bamberg, Goebhardt.

Honneth, A. (2015), Le droit de la liberté, Paris, Gallimard. 
Honneth, A. (2012), Das Recht der Freiheit, Berlin, Suhrkamp.

Honneth, A. (2002), La lutte pour la reconnaissance, Paris, Cerf.

Honneth, A. (1994), Der Kampf um Anerkennung, Francfort-sur-leMain, Suhrkamp.

Honneth, A. (dir.) (1980), Arbeit, Handlung, Normativität, Francfort-sur-le-Main, Suhrkamp.

Kern, H. et Schumann, M. (1984), Das Ende der Arbeitsteilung?, Munich, Beck.

Kern, H. et Schumann, M. (1989), La fin de la division sociale du travail?, Paris, éditions MSH.

Lallement, M. (1996), Sociologie des relations professionnelles, Paris, La Découverte.

Linhart, D. (2015), La comédie du travail, Paris, Érès.

Linhart, Daniele (1991), Le torticolis de l'autruche: l'éternelle modernisation des entreprises françaises, Paris, Seuil.

Marx, Karl (2015), Le chapitre VI du Capital (dit «inédit»), Paris, éditions sociales.

MedA, Dominique (1998), Le travail une valeur en voie de disparition, Paris, Champs Flammarion.

Mercure, Vultur (2010), La signification du travail. Nouveau modèle productif et ethos du travail au Québec, Laval, Presses de l'Université Laval.

Moulier-Boutang, Yann (2007) Le capitalisme cognitif, Paris, éditions Amsterdam.

Naville, Pierre (1957a), De l'aliénation à la jouissance. Genèse de la sociologie du travail chez Marx et Engels, Paris, Anthropos.

Naville, Pierre (1957b), Vers l'automatisme social?, Paris, Gallimard.

Naville, P. (1964), Théorie de l'orientation professionnelle, Paris, Gallimard.

Naville, P. (1984), La Maîtrise du salariat, Paris, Anthropos.

Negt, O. (2007), L'espace public oppositionnel, Paris, Payot.

Negt, O. (2001), Arbeit und menschliche Würde, Göttingen, Steidl.

Negt, O. et Kluge, A. (1981), Geschichte und Eigensinn, Göttingen, Zweitausendeins.

Neumann, Alexander (2015), Après Habermas, Paris, Delga.

Neumann, Alexander (2010), Kritische Arbeitssoziologie, Stuttgart, Schmetterling.

Neumann, Alexander (2011), Review of the Working Time Directive 2003 — Case Study Germany, Economisti associati, Rome.

Rolle, Pierre (1997), Où va le salariat?, Lausanne, éditions Page deux.

Sauer, D. et Wittke, V. (dir.) (2012), Kapitalismustheorie und Arbeit. Neue Ansätze soziologischer Kritik, Campus, Francfort-sur-le-Main.

Scholz, Trebor (2013), Digital Labor. The Internet as Playground and Factory, Londres, Routledge.

Sennett, Richard (2000), Le travail sans qualités, Paris, Albin Michel.

Schumann, Michael (2003), Metamorphosen von Industriearbeit und Arbeiterbewusstsein, Hambourg, VSA. Streeck, W. (2014), Du temps acheté, Paris, Gallimard.

Streeck, W. (1999), Der Korporatismus in Deutschland: zwischen Nationalstaat und europäischer Union, Francfort-sur-le-Main, Campus.

Streeck, W. et Heinze, H. (1999), «Runderneuerung des deutschen Modells. Aufbruch für mehr Jobs», in Arlt/Nehls (dir.) Bündnis für Arbeit, Westdeutscher Verlag.

Thiveaud, J.-M. (1996), «Le risque et son prix: Genèse d'un concept et de son évaluation juridicofinancière (XIV ${ }^{\mathrm{e}}$-XVII ${ }^{\mathrm{e}}$ siècles), in Revue d'économie financière, $\mathrm{n}^{\circ}$ 37, p. 253-274.

Touraine, A. (2005), Un nouveau paradigme, Paris, Fayard.

Touraine, A. (1965), Sociologie de l'action, Paris, Seuil, nouvelle préface de 1999.

Touraine, A. (1966), La conscience ouvrière, Paris, Seuil.

Touraine, A. (1956), L'évolution du travail ouvrier aux usines Renault, Paris, Seuil.

UE (2002), Presidency conclusions — Lisbon European Council, 24 mars 2000.

Vincent, J.-M. (1987), Critique du travail. Le faire et l'agir, Paris, PUF.

Weber, F. (1989), Le travail à-côté, Paris, éditions EHESS.

Wetzel, D. (2015), Arbeit 4.0., Freiburg, Herder. 